\title{
Corpus
}

\section{La Reforma Universitaria y la política al ras del suelo}

Ana Clarisa Agüero

\section{(2) OpenEdition}

\section{Journals}

\section{Electronic version}

URL: http://journals.openedition.org/corpusarchivos/2226

DOI: 10.4000/corpusarchivos. 2226

ISSN: $1853-8037$

\section{Publisher}

Diego Escolar

\section{Electronic reference}

Ana Clarisa Agüero, «La Reforma Universitaria y la política al ras del suelo », Corpus [En línea], Vol. 8, No 1 | 2018, Publicado el 05 julio 2018, consultado el 01 mayo 2019. URL : http://

journals.openedition.org/corpusarchivos/2226 ; DOI : 10.4000/corpusarchivos.2226

This text was automatically generated on 1 May 2019.

Licencia Creative Commons: Atribución-NoComercial 2.5 Argentina (CC BY-NC 2.5 AR) 


\title{
La Reforma Universitaria y la política al ras del suelo
}

\author{
Ana Clarisa Agüero
}

El centenario de la Reforma Universitaria encuentra a la historiografía en una situación diversa a la que marcó los bicentenarios de la Revolución y la Independencia: mientras que aquellos fueron la ocasión de exponer la enorme renovación del conocimiento sobre esos procesos experimentada en los últimos cuarenta años, este es un centenario que, salvo contadas excepciones, no puede reposar sobre una renovación análoga. Pensado en términos de la arena pública más general, eso hace más sencilla la profusión de los usos instrumentales de la reforma y las invocaciones más bien espontáneas o de ocasión. La dimensión política del evento se inflama a la vez que se empobrece, lo que no por habitual es menos problemático.

2 De esa dimensión política de la reforma, interesante e ineludible, a mí me atraen especialmente ciertas cuestiones que he trabajado a nivel local, cordobés, pero estimo ayudarían a reconsiderar otras más generales: la arena tanto social como político-cultural del movimiento universitario, la diversidad de experiencias del proceso, la fisonomía y los tiempos efectivos de los ciclos de politización y radicalización que hicieron que muchos hombres salieran transformados de allí. En este aspecto, si los intentos de releer la experiencia cordobesa desalientan cualquier generalización, subrayando el protagonismo de las realidades locales, el de modificar el ángulo de visión puede ofrecer, creo, pautas de interés más general.

3 Globalmente, me parece que el problema es menos que el evento haya intentado ser remitido a ciertos macrofenómenos que el hecho de que, en general, eso no dialogó con una avanzada analítica orientada a identificar los nexos que se postulaban, lo que hizo que estos funcionaran más como supuestos que como tesis, o se traspolaran formas más o menos canónicas de articular grandes variables (clases medias, generaciones, etc.). En este aspecto, la reforma sigue estando muy precisada de exploraciones más "al ras del suelo", según la feliz expresión de Jacques Revel, y por eso ciertas indicaciones de Gardenia Vidal -que apuntan interrupciones o regresiones en el propio curso de los 
acontecimientos- me parecen una llave muy sugerente para volver a mirar de otra manera cosas que se tenían por dadas.

\section{Primera cuestión: ¿de las aulas a las calles o al revés?}

4 La primera cuestión que me interesa hace a una fórmula de larga vida, propalada por el propio reformismo en momentos muy tempranos: la que señala que ese movimiento fue “de los claustros a las calles". A mi juicio, variar el ángulo de visión permitiría, por un lado, invertir los nexos causales respecto del movimiento en sí mismo y, por otro, reconducir esa imagen a una de sus experiencias, marcada generacionalmente. En el primer aspecto, creo que es posible demostrar que el estallido universitario que suele datarse en junio obedeció a fuerzas sociales y político-culturales muy concretas que lo precedían. Fuerzas de carácter local que, en mayor o menor medida, ya se desplegaban en un espacio público que elaboraban y expandían, como la Asociación Córdoba Libre, los comités georgistas, la actividad seccional de ciertos partidos, las organizaciones obreras y aun el comité antineutralista. La guerra europea, antes que una referencia al hundimiento de Europa, es una vía de movilización y encuentro con las dirigencias italianas; las nuevas inflexiones de la tradición liberal, antes que cuestiones de doctrina, son virajes efectivos en las formaciones que apelan a ella y la declinan en el sentido de cierto progresismo social (por eso su consistencia política pudo ser tanta mientras su anticlericalismo era tan extemporáneo y de combate). Y así.

5 Una mirada tal permite sugerir que el movimiento objetivo va de las calles a las aulas y no al revés, pero también, y este es el segundo aspecto, considerar que si se representa a sí mismo como originario y naciendo en las aulas, es en parte porque para cierta generación lo fue. Los estudiantes, que como señalara Halperin son quienes viven ante todo en un tiempo presente, creen que la política y la movilización han comenzado con ellos. Y dado que, efectivamente, para ellos se abre un universo de acción novedoso, es solo respecto de él que cabe decir que hay una movilización en las aulas que (también o luego) se vuelca a las calles.

\section{Segunda cuestión: las fuerzas reales}

Otra cuestión que me interesa especialmente es la variedad de actores, pertenencias e identidades asociativos, partidarios o de otro orden que atraviesan esa movilización universitaria. Aunque buena parte del estudiantado es independiente, y la vocación de independencia será defendida como condición de la unidad estudiantil, es bastante evidente que muchos forman parte de otras experiencias y tradiciones en el mismo momento en que tiene lugar este proceso, o las abrazan a partir de él; $y$, por ende, que esas otras pertenencias e identidades inciden de diversas formas, sea como esquemas previos de acción y concepción, sea como deliberado intento de sobredeterminar el conflicto, sea en el más espontáneo de comunicar y eslabonar generaciones y orientaciones.

7 Por sus varias formas de presencia y sus compromisos cruzados, de los partidos, quizás el radicalismo sea el que más permite complejizar la trama. Dividido entonces en dos sectores, más y menos vinculados al gobierno provincial (el "azul" y el "rojo", respectivamente), dentro de un sector estudiantil las simpatías están repartidas, en 
especial en la primera fase reformista, cuando el consenso es, al menos en lo relativo a la universidad, muy amplio. Un cierto número de estudiantes ocupa además puestos en la estructura estatal y, al menos en ciertos casos, como el Consejo de Higiene, estos parecen derivar de la proximidad partidaria. A la vez, el hecho de que Yrigoyen intente resolver simultáneamente la cuestión universitaria y la interna local, con vistas a las elecciones provinciales, conducirá en la segunda mitad del año a que una parte muy activa del estudiantado acabe enrolándose en el Centro Liberal Universitario que acompañará la candidatura de su ungido, Elpidio González. Este desplazamiento no corresponde forzosamente a una retracción polémica, ni priva a este sector de experimentar su propia radicalización, pero sí sugiere un encuadramiento que dialoga estrechamente con el curso del proceso universitario y con la mirada positiva de la presencia nacional.

Sin embargo, esa no es entonces la única referencia partidaria. El Partido Demócrata ocupa un lugar relevante en el conflicto, luego bastante desdibujado, en parte por el desinterés de sus sucesores de disputar el legado. Pero si lo que interesa es 1918, su presencia es indiscutible, y se traduce en figuras estudiantiles de cierta gravitación, como Horacio Valdez o Carlos Suárez Pinto, en "amigos mayores" como Amado Roldán y Guillermo Rothe, y aun en el sensible influjo que la antigua propuesta de reformulación universitaria de Ramón J. Cárcano tiene en la primera fase del conflicto. El Partido Socialista, por su parte, tiene una presencia cierta, aunque es preciso aún avanzar en una reconstrucción detallada de su expresión estudiantil. En todo caso, su aval es declarado en las varias visitas nacionales (Mario Bravo, Alfredo Palacios, entonces fuera del partido), sus vacilaciones parecen incidir en las tomas de distancia del yrigoyenismo y su acción se hace sentir también en forma mediada, a través de egresados recientes como Arturo Orgaz o Deodoro Roca, ambos con relaciones próximas aunque no orgánicas (y tampoco lineales, como sugiere el hecho de que Roca sea entonces funcionario del gobierno provincial, en tanto director del Museo Politécnico).

Ciertas experiencias asociativas, que reposan en una larga acumulación característica del fin de siglo y muy bien trabajada por Pablo Vagliente y Gardenia Vidal, son igualmente relevantes para pensar el sustrato de la reforma. Entre ellas sobresale Córdoba Libre, nacida en torno a una suerte de manifiesto librepensador en 1916 y marcadamente politizada a lo largo de 1918. Estímulo decidido del movimiento universitario, el crescendo de agitación que alimenta en la segunda mitad del año será a su vez decisivo en su propia formalización y fijación de estatutos, bajo una junta ejecutiva compuesta por Saúl Taborda, Deodoro Roca y Sebastián Palacio. Córdoba Libre adquiere entonces una marcada heterogeneidad partidaria y social, que llega a expresarse territorialmente en la formación de comités seccionales, y una sensible presencia pública. Su programa reúne motivos reformistas de diversa inspiración, desde aquellos que alimentan un vasto reformismo social anterior hasta los que declinan en sentido georgista, socialista y aun en un municipalismo que merece ser considerado. Allí conviven comerciantes, profesionales, figuras intelectuales de cierto renombre, algún dirigente ferroviario y estudiantes, y no solo es posible señalar las formas concretas de su impulso a la movilización estudiantil sino que también es probable que, para muchos universitarios, esa doble pertenencia tenida o adquirida en el primer umbral de radicalización - haya sido la vía fundamental de una transformación política más duradera. Aun para ciertos referentes de las cohortes anteriores, comenzando por Deodoro Roca, Córdoba Libre, con su crecimiento, agitación y presencia territorial, parece haber actuado como la experiencia decisiva para ir hacia otro lugar. 
10 También la gravitación de otras fuerzas institucionales merece ser considerada para, a contrapelo de la imagen de la "Córdoba católica", exponer cómo funcionó concretamente el intento de la Iglesia de incidir en el conflicto. Tomar como descripciones ciertas las que eran figuras de combate, incluidas las variaciones en torno al jesuitismo, no es la mejor vía, aunque haya sido exitosa. En este punto, una serie de trabajos recientes, como los de Mauro o Schenonne, permite entender mejor el modo en que los sectores católicos orgánicos pasaron del reformismo del primer ciclo al antirreformismo del segundo; cómo eso se enlazaba a sus propias disputas y proyectos (comenzando por el de una universidad católica); y cómo, también, en el plano de la política cruda, creencia y activismo estudiantil podían guardar relaciones muy distintas. Los sobreentendidos sobre la Corda Frates también flaquean con esto, mostrando en cierto modo cómo, bajo los pies de esa identidad declamada, lo único que permanece indiscutible es el juego de los intereses.

11 La cuestión de las fuerzas reales en juego merecería varios desgloses más: el contrapunto entre egresados y estudiantes, en tanto sectores con expectativas y demandas propias, que provocan desencuentros efectivos a lo largo del proceso; el habido entre las generaciones canónicas y las intersticiales (Roca o Martínez Paz frente a Enrique Barros, Ismael Bordabehere $u$ Horacio Valdez); el que une o distancia alternativamente al movimiento estudiantil de experiencias como el antineutralista Comité Pro-Dignidad Nacional.

\section{Tercera cuestión: politización y radicalización, umbrales hacia otra cosa}

12 La tercera cuestión podría ser también la primera: la propia evolución del conflicto universitario en Córdoba sigue precisando una mirada minuciosa, capaz de identificar los momentos de expansión y retracción, de caracterizar las formas de la política en cada caso y de datar con cierta precisión los umbrales de radicalización que acabaron por precipitar el proceso en un sentido. En este punto, es muy importante volver a considerar la primera fase del movimiento, iniciada en diciembre de 1917. Por un lado, porque permite advertir cómo se van agregando las diversas demandas propiamente institucionales; por otro, porque sugiere que el proceso de reformas pudo cerrarse mucho antes, sin dar forzosamente lugar al estallido universitario, que es el evento sobre el que reposa toda la fuerza de la reforma cordobesa como hito fundacional de una o varias tradiciones.

13 Sobre un sustrato de extendida disconformidad ante el estado de la institución universitaria, ciertas medidas puntuales, como el anuncio del cierre del internado de Medicina, agregan demandas y abren el espacio de la protesta. Interrumpido por las vacaciones, en marzo de 1918 el conflicto se reanuda y precipita en el sentido de la huelga, lo que a su vez trae el cierre de la universidad y la llegada de la primera intervención. Hacia finales de mayo, esa intervención ha introducido modificaciones más bien modosas pero que abren la expectativa de una renovación institucional, en gran medida identificada con el nuevo lugar de los consejos directivos electivos en el gobierno de la universidad, en detrimento de las viejas academias vitalicias. Dije que la reforma es modosa porque, como señala Buchbinder, ese orden de medidas había tenido lugar en Buenos Aires doce años antes, pero es indudable que en el cuadro cordobés representaban un cambio auspicioso. La casi universal aceptación de la intervención Matienzo acusa un 
punto muy interesante, porque si se atiende al clima de fines de mayo, la reforma universitaria parece estar ya hecha y encaminada: la elección rectoral vendría a rematar un proceso que se declara prácticamente cerrado desde ese momento, y en esto parece haber un acuerdo muy general.

Lo que surge de esta constatación tan elemental es que ese primer momento es bastante distinto al que lo seguirá desde el mayormente inesperado resultado de la elección rectoral; que no hay una evolución forzosa entre uno y otro; y que, en todo caso, de haber vencido Enrique Martínez Paz, candidato reformista, todo pudo haberse detenido allí. Sin abundar en las vías alternativas, una resolución de ese orden hubiera extinguido las condiciones eficientes del estallido tal como lo conocemos, y variado las de sus consecuencias, comenzando por el creciente componente social de la reforma, precipitado desde entonces y en conexión con la agitación general. En cambio, al tiempo confiado de fines de mayo siguió el tiempo exasperado del resultado electoral, y tomar seriamente este momento, de una renovada, virulenta y muy compleja politización, que se radicalizó al compás de la ruptura del frente reformista, parece fundamental para entender cuándo, de qué modo y por qué, el movimiento de reforma universitaria pasó a ser otra cosa.

así, sin duda junio inicia un ciclo novedoso de politización, que asume un cariz más radical que el anterior también en la medida que una parte del movimiento, que incluye a los católicos orgánicos y a ciertos sectores "liberales", ha quedado escindida y enfrentada a la Federación Universitaria. Este segundo ciclo hace su propia crispación y la segunda intervención trae una segunda paz, a la que ya no se llega igual, y esto no solo porque el ciclo de movilización ha sido más virulento y sostenido, ha dado lugar a una intensa acción territorial que estimuló el vínculo con otras realidades y asume una declinación más abiertamente democrática y social, sino, también, porque esa paz llega acompañada de un intenso sentimiento de triunfo. Sobre el cierre de ese ciclo, el ataque a Enrique Barros, que es lo que más que nunca precipita el acompañamiento sindical, acabará representando un segundo umbral de radicalización que solo algunos podrán transitar. Un par de ejemplos pueden ilustrar esto. Ismael Bordabehere, que en noviembre se integrará al Comité Liberal Universitario, dirige los primeros números de La Gaceta Universitaria en 1919, salidos a mediados de año. A esa altura, su voz ya ha sonado en la denuncia de los sucesos de la Semana Trágica, otra inflexión traída por enero, y su prédica adquiere un indiscutible tono obrerista, que marca la fase más radicalizada de la revista. Hay constancia de que Bordabehere busca deliberadamente inclinar el movimiento hacia los sectores trabajadores y crear un lectorado obrero para La Gaceta. Sin embargo, su opción ha sido acompañar el voto a González, candidato yrigoyenista finalmente derrotado. El caso es atractivo, porque permite asomarse a un viraje más general y avizorar las propias apuestas obreras de un sector del radicalismo, alimentado por la integración de jóvenes hasta allí independientes, que no desdeñan críticas al capitalismo o las fuerzas represivas. En fluido contacto con Gregorio Bermann, Bordabehere despliega su mirada en un lenguaje muy semejante el del responsable de las asociaciones culturales, aunque es presumible que no siempre aludan a las mismas cosas (y que, en el caso de Bordabehere, el viraje llegue hasta ahí).

Otro ejemplo: ni Ceferino Garzón Maceda ni Roca son en marzo de 1918 los que serán en Mente, la revista que en 1920 reúne una serie de intervenciones de filobolcheviques a libertarias, en la que también se expresan miradas críticas y desafíos al movimiento reformista, desde un casi fuera de campo que enarbola las promesas de la revolución 
verdadera frente a los límites de la reforma. En quienes serán tan protagónicos en la elaboración del reformismo como tradición, a lo largo de las décadas, sin duda adquiere especial interés comprender cuándo, cómo y por qué se fue operando ese desplazamiento. Y aquí el problema dista de ser local aunque admita muchas expresiones intermedias, entre las que me inclino a ubicar a esas dos figuras. En definitiva, se trata de entender en qué momento alguien que cree que hay que iluminar al obrero pasa a considerar que debe compartir trincheras con él; o alguien que considera que el horizonte es la libertad republicana pasa a conceder, al menos fugazmente, que Rusia puede ofrecer un norte para más que sí misma (y con esto se aleja del gran consenso respecto del valor histórico de su revolución que marca al liberalismo constitucional).

Un contexto muy general es el que ofrece el vasto acuerdo en torno a la "justicia social" como correlato de la democratización política; acuerdo que, según Halperin, marca la etapa 1917-1922 y, podría decirse, desplaza todo el arco político hacia la izquierda. Pero es evidente que ese sustrato, que abonó las formas progresivas de la política en que el movimiento universitario nació, no fue lo que definió su fisonomía efectiva, que en parte se precisa en su propia dinámica política, con sus regularidades y azares. Allí parece residir el deslizamiento que permitió que muchos viraran hacia posturas reformistaradicales o revolucionarias, que se transformaran durablemente a sí mismos (algunos, simplemente, rasgando su piel criolla) y que procuraran, a lo largo de los años, regular las lecturas de un proceso que tendía a licuarse o inflamarse conforme las coyunturas y tomas de posición ulteriores.

\section{BIBLIOGRAPHY}

Agüero, A. C. (e/p). Estudiantes reformistas. Notas sobre la experiencia, las generaciones y las ideas (1880/1935). En P. Buchbinder (Comp.). Juventudes Universitarias en América Latina: ayer y hoy. Rosario: UNR Editora.

Agüero, A. C. (2016). Córdoba. 1918, más acá de la reforma". En A Gorelik, y F. Arêas Peixoto (Comps.), Ciudades sudamericanas como arenas culturales. Buenos Aires: Siglo XXI Editores.

Agüero, A. C. y Núñez, M. V. (2018). Los asesinos de Barros. Una pesquisa sobre la derrota. En D. Mauro, y J. Zanca (Comps.), La reforma universitaria cuestionada. Rosario: Fhumyar Ediciones.

Buchbinder, P. (2005). Historia de las Universidades Argentinas. Buenos Aires: Editorial Sudamericana.

Cárcano, R. J. (1892). Universidad de Córdoba. Algunas palabras sobre su organización. Buenos Aires: Félix Lajouane.

Di Filippo, L. (1920). A definirse. Mente. Publicación de crítica social, Año I, № 2.

Domínguez Rubio, L. (2016) Mente. Publicación de crítica social. En http:// culturasinteriores.ffyh.unc.edu.ar/iec002.jsp?pidf=2Z6WFNDIP\&po=R .

Halperin Donghi, T. (1999). Estudio preliminar. En Vida y muerte de la república verdadera (1910-1930). Buenos Aires: Ariel. 
Julliard, J. (2012). Verité du réformisme. Mil neuf cent. Revue d'histoire Intellectuelle, № 30. Mauro, D. (2018). Los católicos frente a la reforma universitaria (1917-1922). En D. Mauro y J. Zanca (Comps.), La reforma universitaria cuestionada. Rosario: Fhumyar Ediciones.

Prochasson, Ch. (2012). Nouveaux regards sur le réformisme. Mil neuf cent. Revue d'histoire Intellectuelle, № 30 .

Schenone, G. (2011). El accionar del estudiantado católico en la UNC durante la Reforma universitaria de 1918. Modernidades, № 11.

Vagliente, P. (2015). Asociativa, movilizada, violenta. La vida pública en Córdoba, 1850-1930. Villa María: EDUVIM (2 T.).

Vidal, G. (2007). La reforma universitaria de 1918 y su repercusión en los resultados electorales. En G. Vidal, (Comp.), La política y la gente. Estudios sobre modernidad y espacio público. Córdoba, 1880-1960. Córdoba: Ferreyra Editor.

Vidal, G. El asociacionismo laicista y la reforma universitaria de 1918 (Córdoba-Argentina). En www.fee.tche.br/sitefee/download/jornadas/2/h1-02.pdf .

\section{AUTHOR}

\section{ANA CLARISA AGÜERO}

Universidad Nacional de Córdoba y Consejo Nacional de Investigaciones Científicas y

Tecnológicas, Argentina

Correo electrónico: anaclarisaa@yahoo.es 